IJMMS 26:10 (2001) 581-588

PII. S0161171201020087

http://ijmms.hindawi.com

(C) Hindawi Publishing Corp.

\title{
ON BLOCKERS IN BOUNDED POSETS
}

\author{
ANDREY O. MATVEEV \\ (Received 15 November 2000 and in revised form 24 May 2001)
}

\begin{abstract}
Antichains of a finite bounded poset are assigned antichains playing a role analogous to that played by blockers in the Boolean lattice of all subsets of a finite set. Some properties of lattices of generalized blockers are discussed.
\end{abstract}

2000 Mathematics Subject Classification. 06A06, 90C27.

1. Introduction. Blocking sets for finite families of finite sets are important objects of discrete mathematics (see [5, Chapter 8] and [3]).

A set $H$ is called a blocking set for a nonempty family $\mathscr{G}=\left\{G_{1}, \ldots, G_{m}\right\}$ of nonempty subsets of a finite set if for each $k \in\{1, \ldots, m\}$ we have $\left|H \cap G_{k}\right| \geq 1$. The blocker of $\mathscr{G}$ is the family of all inclusion-wise minimal blocking sets for $\mathscr{G}$.

A family of subsets of a finite set is called a clutter (or a Sperner family) if no set from it contains another. If the family is empty or if it consists of only one subset, $\{\varnothing\}$, then the corresponding clutter is called trivial.

The concepts of blocker map and complementary map on clutters [1] made it possible to clarify the relationship between specific families of sets, arising from the matroid theory, and maps on them. The blocker map, that assigns the blocker to a clutter, is defined on all clutters, including trivial clutters.

The following property $[2,6]$ is basic: for a clutter $\mathscr{G}$, the blocker of its blocker coincides with $\varphi$.

We show that the concepts of blocking set and blocker can be extended when passing from discussing clutters, considered as antichains of the Boolean lattice of all subsets of a finite set, to exploring antichains of arbitrary finite bounded posets (a poset $P$ is called bounded if it has a unique minimal element, denoted $\hat{0}_{P}$, and a unique maximal element, denoted $\hat{1}_{P}$ ).

In Section 2, the notion of intersecter plays a role analogous to that played by the notion of blocking set in the Boolean lattice of all subsets of a finite set. In Section 3, we explore the structure of subposets of intersecters in Cartesian products of posets. In Section 4, some properties of the blocker map and complementary map are shortly discussed. In Section 5, the structure of lattices of generalized blockers is reviewed.

2. Intersecters and complementers. We refer the reader to [7, Chapter 3] for basic information and terminology in the theory of posets.

For a poset $Q, Q^{\text {a }}$ denotes its atom $\operatorname{set} ; \min Q$ and $\max Q$ denote the sets of all minimal elements and all maximal elements of $Q$, respectively; $\mathfrak{I}_{Q}(X)$ and $\mathfrak{f}_{Q}(X)$ denote the order ideal and order filter of $Q$ generated by a subset $X \subseteq Q$, respectively. If $x, y$ 
are elements of $Q$ and $x<y$ (or $x \leq y$ ), then we write $x<_{Q} y$ (or $x \leq_{Q} y$ ). In a similar way, we denote by $\vee_{Q}$ the operation of join in a join-semilattice $Q$, and we denote by $\wedge_{Q}$ the operation of meet in a meet-semilattice $Q$. We use $\times$ to denote the operation of Cartesian product of posets.

For a finite family $\varphi$ of finite sets, its conventional blocker is denoted by $\mathscr{B}(\mathscr{G})$.

Throughout $P$ stands for a finite bounded poset with $|P|>1$. We start with extending the concept of blocking set.

DEFINITION 2.1. Let $A$ be a subset of $P$.

- If $A \neq \varnothing$ and $A \neq\left\{\hat{0}_{P}\right\}$, then an element $b \in P$ is an intersecter for $A$ in $P$ if for every $a \in A-\left\{\hat{0}_{P}\right\}$, we have

$$
\left|\mathfrak{I}_{P}(b) \cap \mathfrak{I}_{P}(a) \cap P^{\mathrm{a}}\right| \geq 1 .
$$

- If $A=\left\{\hat{0}_{P}\right\}$ then $A$ has no intersecters in $P$.

- If $A=\varnothing$ then every element of $P$ is an intersecter for $A$ in $P$.

- Every non-intersecter for $A$ in $P$ is a complementer for $A$ in $P$.

Let $\mathscr{L}$ denote a finite Boolean lattice. If $A$ is a nonempty subset of the poset $\mathscr{L}-\left\{\hat{0}_{\mathscr{L}}\right\}$, then an element $b \in \mathscr{L}$ is an intersecter for $A$ in $\mathscr{L}$ if and only if $\tilde{I}_{\mathscr{L}}(b) \cap \mathscr{L}^{\text {a }}$ is a blocking set for the family $\left\{\mathfrak{z}_{\mathscr{L}}(a) \cap \mathscr{L}^{\mathrm{a}}: a \in A\right\}$.

We denote by $\mathbf{I}(P, A)$ and $\mathbf{C}(P, A)$ the sets of all intersecters and all complementers for $A$ in $P$, respectively. We consider the sets $\mathbf{I}(P, A)$ and $\mathbf{C}(P, A)$ as subposets of the poset $P$. For a one-element set $\{a\}$ we write $\mathbf{I}(P, a)$ instead of $\mathbf{I}(P,\{a\})$ and $\mathbf{C}(P, a)$ instead of $\mathrm{C}(P,\{a\})$.

We have the partition $\mathbf{I}(P, A) \dot{\cup} \mathbf{C}(P, A)=P$. For a nonempty subset $A \subseteq P-\left\{\hat{0}_{P}\right\}$, the subposets of all its intersecters and complementers are nonempty; indeed, we have $\mathbf{I}(P, A) \ni \hat{1}_{P}$ and $\mathbf{C}(P, A) \ni \hat{0}_{P}$. It follows from Definition 2.1 that for such a subset $A$, we have

$$
\mathbf{I}(P, A)=\mathbf{I}(P, \min A), \quad \mathbf{C}(P, A)=\mathbf{C}(P, \min A),
$$

therefore, in most cases, we may restrict ourselves to considering intersecters and complementers for antichains; further,

$$
\mathbf{I}(P, A)=\bigcap_{a \in A} \mathbf{I}(P, a), \quad \mathbf{C}(P, A)=\bigcup_{a \in A} \mathbf{C}(P, a) .
$$

For all antichains (including the empty antichain) $A_{1}, A_{2}$ of $P$ with $\mathfrak{f}_{P}\left(A_{1}\right) \subseteq \mathfrak{f}_{P}\left(A_{2}\right)$, we have

$$
\mathbf{I}\left(P, A_{1}\right) \supseteq \mathbf{I}\left(P, A_{2}\right), \quad \mathbf{C}\left(P, A_{1}\right) \subseteq \mathbf{C}\left(P, A_{2}\right) .
$$

Clearly, the subposet $\mathbf{I}(P, a)$ of all intersecters for an element $a \in P$ is the order filter $\mathfrak{f}_{P}\left(\mathfrak{I}_{P}(a) \cap P^{\mathrm{a}}\right)$, hence, in view of (2.3), equality (2.5) in the following lemma holds.

LEMMA 2.2. Let $A$ be a nonempty subset of $P-\left\{\hat{0}_{P}\right\}$. The subposet of all intersecters for $A$ in $P$ is determined by the following equivalent equalities:

$$
\mathbf{I}(P, A)=\bigcap_{a \in A} \mathfrak{f}_{P}\left(\mathfrak{I}_{P}(a) \cap P^{\mathrm{a}}\right),
$$




$$
\mathbf{I}(P, A)=\bigcup_{E \in \mathscr{B}\left(\left\{\tilde{I}_{P}(a) \cap P^{\mathrm{a}}: a \in A\right\}\right)} \bigcap_{e \in E} \mathfrak{f}_{P}(e) .
$$

Proof. To prove (2.6), note that the inclusion

$$
\mathbf{I}(P, A) \supseteq \bigcup_{E \in \mathscr{B}\left(\left\{\tilde{I}_{P}(a) \cap P^{\mathrm{a}}: a \in A\right\}\right)} \bigcap_{e \in E} \mathfrak{f}_{P}(e)
$$

follows from the definition of intersecters.

We are left with proving the inclusion

$$
\mathbf{I}(P, A) \subseteq \bigcup_{E \in \mathscr{B}\left(\left\{\tilde{I}_{P}(a) \cap P^{\mathrm{a}}: a \in A\right\}\right)} \bigcap_{e \in E} \mathfrak{f}_{P}(e) .
$$

Assume that it does not hold, and consider such an intersecter $b$ for $A$ that $b \notin$ $\bigcup_{E \in \mathscr{B}\left(\left\{\mathfrak{I}_{P}(a) \cap P^{\mathrm{a}}: a \in A\right\}\right)} \bigcap_{e \in E} \mathfrak{f}_{P}(e)$. In this case, the inclusion $b \in \bigcap_{e \in E} \mathfrak{f}_{P}(e)$ holds not for all sets $E$ from the family $\mathscr{B}\left(\left\{I_{P}(a) \cap P^{\mathrm{a}}: a \in A\right\}\right)$, hence there exists such an element $a \in A$ that $\left|\mathfrak{I}_{P}(b) \cap \mathfrak{I}_{P}(a) \cap P^{\mathrm{a}}\right|=0$. Therefore $b$ is not an intersecter for $A$, but this contradicts our choice of $b$. Hence, (2.6) holds.

Thus, for every subset $A$ of the poset $P$, the subposet of all intersecters for $A$ in $P$ is an order filter of $P$, that is, $\mathbf{I}(P, A)=\mathfrak{f}_{P}(\min \mathbf{I}(P, A))$. As a consequence, the subposet $\mathbf{C}(P, A)$ of all complementers for $A$ in $P$ is the order ideal $\mathfrak{z}_{P}(\max \mathbf{C}(P, A))$.

If $A$ is a subset of the poset $P$ then we call the antichain $\min \mathbf{I}(P, A)$ the blocker of $A$ in $P$. We call elements of the blocker $\min \mathbf{I}(P, A)$ minimal intersecters for $A$ in $P$, and we call elements of the antichain $\max \mathbf{C}(P, A)$ maximal complementers for $A$ in $P$.

The images of intersecters under suitable order-preserving maps are also intersecters.

Proposition 2.3. Let $P_{1}$ and $P_{2}$ be disjoint finite bounded posets with $\left|P_{1}\right|,\left|P_{2}\right|>1$. Let $\psi: P_{1} \rightarrow P_{2}$ be an order-preserving map such that

$$
\psi\left(\hat{0}_{P_{1}}\right)=\hat{0}_{P_{2}}, \quad \psi\left(x_{1}\right)>_{P_{2}} \hat{0}_{P_{2}}, \quad \forall x_{1}>_{P_{1}} \hat{0}_{P_{1}} .
$$

For every subset $A_{1}$ of $P_{1}$

$$
\psi\left(\mathbf{I}\left(P_{1}, A_{1}\right)\right) \subseteq \mathbf{I}\left(P_{2}, \psi\left(A_{1}\right)\right) .
$$

Proof. There is nothing to prove for $A_{1}=\varnothing \subset P$ and $A_{1}=\left\{\hat{0}_{P_{1}}\right\}$. So suppose that $A_{1} \neq \varnothing \subset P$ and $A_{1} \neq\left\{\hat{0}_{P_{1}}\right\}$. Let $b_{1}$ be an intersecter for $A_{1}$. According to Definition 2.1, for all $a_{1} \in A_{1}, a_{1}>_{P_{1}} \hat{0}_{P_{1}}$, we have $\left|\mathfrak{I}_{P_{1}}\left(b_{1}\right) \cap \mathfrak{I}_{P_{1}}\left(a_{1}\right) \cap P_{1}{ }^{\mathrm{a}}\right| \geq 1$, and in view of (2.9), for every atom $z_{1} \in \mathfrak{I}_{P_{1}}\left(b_{1}\right) \cap \mathfrak{I}_{P_{1}}\left(a_{1}\right) \cap P_{1}{ }^{a}$ we have the inclusion

$$
\mathfrak{I}_{P_{2}}\left(\psi\left(z_{1}\right)\right) \cap P_{2}{ }^{\mathrm{a}} \subseteq \mathfrak{I}_{P_{2}}\left(\psi\left(a_{1}\right)\right) \cap P_{2}{ }^{\mathrm{a}},
$$

the left-hand part of which is nonempty. Hence, for all $a_{2} \in \psi\left(A_{1}\right)$ the inclusion $b_{1} \in$ I $\left(P, A_{1}\right)$ implies that

$$
\left|\mathfrak{I}_{P_{2}}\left(\psi\left(b_{1}\right)\right) \cap \mathfrak{I}_{P_{2}}\left(\psi\left(a_{1}\right)\right) \cap P_{2}{ }^{\mathrm{a}}\right| \geq 1 .
$$

This means that $\psi\left(b_{1}\right) \in \mathbf{I}\left(P_{2}, \psi\left(A_{1}\right)\right)$ and completes the proof. 
3. Intersecters in Cartesian products of posets. In this section, we study the structure of subposets of intersecters in Cartesian products of two finite posets.

Proposition 3.1. Let $P_{1}$ and $P_{2}$ be disjoint finite bounded posets with $\left|P_{1}\right|,\left|P_{2}\right|>2$. Let $Q$ denote the poset

$$
\left(P_{1}-\left\{\hat{0}_{P_{1}}, \hat{1}_{P_{1}}\right\}\right) \times\left(P_{2}-\left\{\hat{0}_{P_{2}}, \hat{1}_{P_{2}}\right\}\right) \dot{\cup}\left\{\hat{0}_{Q}, \hat{1}_{Q}\right\},
$$

where $\hat{0}_{Q}$ and $\hat{1}_{Q}$ are the adjoint new least and greatest elements. Let $A$ be a nonempty subset of the poset $Q-\left\{\hat{0}_{Q}, \hat{1}_{Q}\right\}$, and let $\left.A\right|_{P_{1}}$ and $\left.A\right|_{P_{2}}$ denote the subsets $\left\{a_{1} \in P_{1}\right.$ : $\left.\left(a_{1} ; a_{2}\right) \in A\right\}$ and $\left\{a_{2} \in P_{2}:\left(a_{1} ; a_{2}\right) \in A\right\}$, respectively.

(i) If $\min \mathbf{I}\left(P_{1},\left.A\right|_{P_{1}}\right)=\left\{\hat{1}_{P_{1}}\right\}$ or $\min \mathbf{I}\left(P_{2},\left.A\right|_{P_{2}}\right)=\left\{\hat{1}_{P_{2}}\right\}$, then

$$
\mathbf{I}(Q, A)=\min \mathbf{I}(Q, A)=\left\{\hat{1}_{Q}\right\} .
$$

(ii) If $\min \mathbf{I}\left(P_{1},\left.A\right|_{P_{1}}\right) \neq\left\{\hat{1}_{P_{1}}\right\}$ and $\min \mathbf{I}\left(P_{2},\left.A\right|_{P_{2}}\right) \neq\left\{\hat{1}_{P_{2}}\right\}$, then

$$
\mathbf{I}(Q, A)=\left(\mathbf{I}\left(P_{1},\left.A\right|_{P_{1}}\right)-\left\{\hat{1}_{P_{1}}\right\}\right) \times\left(\mathbf{I}\left(P_{2},\left.A\right|_{P_{2}}\right)-\left\{\hat{1}_{P_{2}}\right\}\right) \dot{\cup}\left\{\hat{1}_{Q}\right\},
$$

and $\min \mathbf{I}(Q, A)=\min \mathbf{I}\left(P_{1},\left.A\right|_{P_{1}}\right) \times \min \mathbf{I}\left(P_{2},\left.A\right|_{P_{2}}\right)$.

Proof. The atom set $Q^{\mathrm{a}}$ of the poset $Q$ is $P_{1}{ }^{\mathrm{a}} \times P_{2}{ }^{\mathrm{a}}$, therefore, by (2.5), the subposet of intersecters for $A$ in $Q$ is

$$
\begin{aligned}
\mathbf{I}(Q, A) & =\bigcap_{\left(a_{1} ; a_{2}\right) \in A} \mathfrak{f}_{Q}\left(\left(\mathfrak{I}_{P_{1}}\left(a_{1}\right) \times \mathfrak{I}_{P_{2}}\left(a_{2}\right)\right) \cap\left(P_{1}{ }^{\mathrm{a}} \times P_{2}{ }^{\mathrm{a}}\right)\right) \\
& =\left(\mathbf{I}\left(P_{1},\left.A\right|_{P_{1}}\right)-\left\{\hat{1}_{P_{1}}\right\}\right) \times\left(\mathbf{I}\left(P_{2}, A \downarrow_{P_{2}}\right)-\left\{\hat{1}_{P_{2}}\right\}\right) \dot{\cup}\left\{\hat{1}_{Q}\right\},
\end{aligned}
$$

and the statement follows.

Proposition 3.2. Let $P_{1}$ and $P_{2}$ be disjoint finite bounded posets with $\left|P_{1}\right|,\left|P_{2}\right|>1$. Let $Q$ denote the poset $P_{1} \times P_{2}$, and let $A$ be a nonempty subset of the poset $Q-\left\{\hat{0}_{Q}\right\}$. Then

$$
\mathbf{I}(Q, A)=\bigcap_{\left(a_{1} ; a_{2}\right) \in A}\left(\left(P_{1} \times \mathbf{I}\left(P_{2}, a_{2}\right)\right) \cup\left(\mathbf{I}\left(P_{1}, a_{1}\right) \times P_{2}\right)\right) .
$$

Proof. Since the atom set $Q^{\mathrm{a}}$ of the poset $Q$ is $\left(\left\{\hat{\mathrm{O}}_{1}\right\} \times P_{2}{ }^{\mathrm{a}}\right) \dot{\cup}\left(P_{1}^{\mathrm{a}} \times\left\{\hat{\mathrm{O}}_{2}\right\}\right)$, we have, according to equality (2.5),

$$
\begin{aligned}
\mathbf{I}(Q, A) & =\bigcap_{\left(a_{1} ; a_{2}\right) \in A} \mathfrak{f}_{Q}\left(\left(\mathfrak{I}_{P_{1}}\left(a_{1}\right) \times \mathfrak{I}_{P_{2}}\left(a_{2}\right)\right) \cap\left(\left(\left\{\hat{0}_{1}\right\} \times P_{2}{ }^{\mathrm{a}}\right) \dot{\cup}\left(P_{1}{ }^{\mathrm{a}} \times\left\{\hat{0}_{2}\right\}\right)\right)\right) \\
& =\bigcap_{\left(a_{1} ; a_{2}\right) \in A} \mathfrak{f}_{Q}\left(\left(\left\{\hat{0}_{1}\right\} \times\left(\mathfrak{I}_{P_{2}}\left(a_{2}\right) \cap P_{2}{ }^{\mathrm{a}}\right)\right) \dot{\cup}\left(\left(\mathfrak{I}_{P_{1}}\left(a_{1}\right) \cap P_{1}{ }^{\mathrm{a}}\right) \times\left\{\hat{0}_{2}\right\}\right)\right),
\end{aligned}
$$

and the statement follows.

4. Blocker map and complementary map. Let $\mathscr{F}(P)$ denote the distributive lattice of all order filters (partially ordered by inclusion) of $P$, and let $\mathcal{A}(P)$ denote the lattice 
of all antichains of $P$. For antichains $A_{1}, A_{2} \in \mathcal{A}(P)$, we set

$$
A_{1} \leq \mathfrak{A}(P) A_{2} \quad \text { iff } \mathfrak{f}_{P}\left(A_{1}\right) \subseteq \mathfrak{f}_{P}\left(A_{2}\right) ;
$$

in other words, we make use of the isomorphism $\mathscr{F}(P) \rightarrow \mathcal{H}(P): F \mapsto \min F$. We call the least element $\hat{0}_{\mathcal{H}(P)}=\varnothing \subset P$ and greatest element $\hat{1}_{\mathcal{A}(P)}=\left\{\hat{0}_{P}\right\}$ of the lattice $\mathcal{A}(P)$ the trivial antichains of $P$. They are counterparts of trivial clutters.

Recall (cf. [4]) that for $A_{1}, A_{2} \in \mathfrak{A}(P)$,

$$
A_{1} \vee_{\mathfrak{A}(P)} A_{2}=\min \left(A_{1} \cup A_{2}\right), \quad A_{1} \wedge_{\mathfrak{H}(P)} A_{2}=\min \left(\mathfrak{f}_{P}\left(A_{1}\right) \cap \mathfrak{f}_{P}\left(A_{2}\right)\right) .
$$

Let $6: \mathcal{A}(P) \rightarrow \mathcal{A}(P)$ be the blocker map on $\mathcal{A}(P)$; by definition,

$$
6: A \longmapsto \min \mathbf{I}(P, A) .
$$

In particular, for every $a \in P, a>_{P} \hat{0}_{P}$, we have $\mathfrak{b}(\{a\})=\mathfrak{I}_{P}(a) \cap P^{\mathrm{a}}$. We also have

$$
\mathfrak{b}(\varnothing \subset P)=\left\{\hat{0}_{P}\right\}, \quad \mathfrak{b}\left(\left\{\hat{0}_{P}\right\}\right)=\varnothing \subset P .
$$

For a one-element antichain $\{a\}$, we write $\mathfrak{b}(a)$ instead of $\mathfrak{b}(\{a\})$.

If $A$ is a nontrivial antichain of $P$ then Lemma 2.2 implicitly states the following equalities in $\mathcal{A}(P)$ :

$$
\mathfrak{b}(A)=\bigwedge_{a \in A} \bigvee_{e \in \mathfrak{b}(a)}\{e\}=\bigvee_{E \in \mathscr{B}(\{\mathfrak{b}(a): a \in A\})} \bigwedge_{e \in E}\{e\}
$$

Let $\mathfrak{2}(P)$ denote the image of $\mathfrak{A}(P)$ under the blocker map. The set $\mathfrak{B}(P)$ is equipped, by definition, with the partial order induced by the partial order on $\mathcal{A}(P)$. For a blocker $B \in \mathfrak{B}(P)$, the subposet $\mathfrak{b}^{-1}(B)=\{A \in \mathfrak{A}(P): \mathfrak{b}(A)=B\}$ is the preimage of $B$ under the blocker map.

The following lemma is a reformulation of (2.4).

LEMMA 4.1. If $A_{1}, A_{2} \in \mathfrak{A}(P)$ and $A_{1} \leq_{\mathcal{H}(P)} A_{2}$ then $\mathfrak{b}\left(A_{1}\right) \geq_{\mathfrak{B}(P)} \mathfrak{b}\left(A_{2}\right)$.

Definition 2.1 implies the following reciprocity property for intersecters: for every antichain $A$ of $P$, we have

$$
A \subseteq \mathbf{I}(P, \mathfrak{b}(A)) .
$$

In the theory of blocking sets the following fact is basic.

Proposition 4.2 (see $[2,6])$. For any clutter $\mathscr{G}, \mathscr{B}(\mathscr{B}(\mathscr{G}))=\mathscr{G}$.

This statement may be generalized in the following way.

THEOREM 4.3. The restriction map $\left.\mathfrak{b}\right|_{\mathfrak{B}(P)}$ is an involution, that is, for each blocker $B \in \mathfrak{B}(P), \mathfrak{b}(\mathfrak{b}(B))=B$.

Proof. There is nothing to prove for the trivial blockers $B=\hat{0}_{\mathfrak{B}(P)}=\varnothing \subset P$ and $B=\hat{1}_{\mathfrak{B}(P)}=\left\{\hat{0}_{P}\right\}$. So suppose that $B$ is nontrivial. Choose an arbitrary antichain $A^{\prime} \in$ $\mathfrak{b}^{-1}(B)$. With regard to reciprocity property for intersecters, every element of $A^{\prime}$ is an intersecter for the antichain $B=\mathfrak{b}\left(A^{\prime}\right)$. In other words, for each element $a^{\prime} \in A^{\prime}$ we 
have the inclusion $a^{\prime} \in \mathbf{I}(P, B)=\bigcap_{b \in B} \mathfrak{f}_{P}(\mathfrak{b}(b))$. Taking this inclusion into account, we assign to the antichain $A^{\prime}$ the antichain

$$
A=\min \bigcap_{b \in B} \mathfrak{f}_{P}(\mathfrak{b}(b)) \in \mathfrak{b}^{-1}(B),
$$

which is the blocker of $B$, by (2.5). Then $\mathfrak{b}(A)=B, \mathfrak{b}(B)=A$, and the theorem follows.

By Lemma 2.2, a nontrivial antichain $A$ of $P$, considered as an element of $\mathcal{A}(P)$, is a fixed point of the blocker map on $\mathcal{H}(P)$ if and only if $A=\wedge_{a \in A} \vee_{e \in \mathfrak{b}(a)}\{e\}$ or, equivalently, $A=\vee_{E \in \mathscr{B}(\{\mathfrak{b}(a): a \in A\})} \wedge_{e \in E}\{e\}$. We study the structure of a preimage of the blocker map.

THEOREM 4.4. For each blocker $B \in \mathfrak{B}(P)$, its preimage $\mathfrak{b}^{-1}(B)$ is a join-subsemilattice of the lattice $\mathcal{A}(P)$.

Proof. There is nothing to prove for a trivial blocker $B$, so suppose that $B$ is nontrivial. Choose two antichains $A_{1}, A_{2} \in \mathfrak{b}^{-1}(B)$. According to (4.5), we have the following equalities in the lattice $\mathcal{A}(P)$ :

$$
B=\mathfrak{b}\left(A_{1}\right)=\bigwedge_{a_{1} \in A_{1}} \bigvee_{e \in \mathfrak{b}\left(a_{1}\right)}\{e\}=\mathfrak{b}\left(A_{2}\right)=\bigwedge_{a_{2} \in A_{2}} \bigvee_{e \in \mathfrak{b}\left(a_{2}\right)}\{e\}
$$

Therefore

$$
B=\bigwedge_{a \in A_{1} \vee_{\mathcal{H}(P)} A_{2}} \bigvee_{e \in \mathfrak{b}(a)}\{e\}=\mathfrak{b}\left(A_{1} \vee_{\mathcal{H}(P)} A_{2}\right)
$$

Hence $A_{1} \vee_{\mathfrak{A}(P)} A_{2} \in \mathfrak{b}^{-1}(B)$.

The greatest element of $\mathfrak{b}^{-1}(B)$ is $\mathfrak{b}(B)$.

Let $\mathfrak{c}: \mathcal{A}(P) \rightarrow \mathcal{A}(P)$ be the complementary map on $\mathcal{A}(P)$; by definition,

$$
\mathfrak{c}: A \longmapsto \max \mathbf{C}(P, A) .
$$

In particular, we have $\mathfrak{c}(\varnothing \subset P)=\varnothing \subset P$ and $\mathfrak{c}\left(\left\{\hat{0}_{P}\right\}\right)=\left\{\hat{1}_{P}\right\}$.

Let $\mathfrak{C}(P)$ denote the image of $\mathfrak{A}(P)$ under the complementary map. The set $\mathfrak{C}(P)$ is equipped, by definition, with the partial order induced by the partial order on the distributive lattice of order ideals of $P$ : for $C_{1}, C_{2} \in \mathfrak{C}(P)$, we set $C_{1} \leq \mathfrak{C}(P) C_{2}$ if and only if $\mathfrak{I}_{P}\left(C_{1}\right) \subseteq \mathfrak{I}_{P}\left(C_{2}\right)$.

5. Lattice of blockers. In this section, we study the structure of the poset of blockers in $P$.

LEMMA 5.1. The poset $23(P)$ of blockers in $P$ is a meet-subsemilattice of the lattice $\mathcal{A}(P)$.

Proof. We have to prove that for all $B_{1}, B_{2} \in \mathfrak{Z}(P)$, it holds $B_{1} \wedge_{\mathfrak{H}(P)} B_{2} \in \mathfrak{2} \mathfrak{B}(P)$. There is nothing to prove when one of the blockers $B_{1}, B_{2}$ is trivial. Suppose that both $B_{1}$ and $B_{2}$ are nontrivial. With the help of Theorem 4.3 , we write

$$
B_{1} \wedge_{\mathfrak{H}(P)} B_{2}=\mathfrak{b}\left(\mathfrak{b}\left(B_{1}\right)\right) \wedge_{\mathfrak{A}(P)} \mathfrak{b}\left(\mathfrak{b}\left(B_{2}\right)\right) .
$$


According to (4.5), we have the following equalities in $\mathcal{A}(P)$ :

$$
\begin{aligned}
& B_{1} \wedge \mathfrak{H}(P) B_{2}=\left(\bigwedge_{a_{1} \in \mathfrak{b}\left(B_{1}\right)} \bigvee_{e \in \mathfrak{b}\left(a_{1}\right)}\{e\}\right) \wedge_{\mathfrak{H}(P)}\left(\bigwedge_{a_{2} \in \mathfrak{b}\left(B_{2}\right)} \bigvee_{e \in \mathfrak{b}\left(a_{2}\right)}\{e\}\right) \\
& =\bigwedge_{a \in \mathfrak{b}\left(B_{1}\right) \vee_{\mathfrak{H}(P)} \mathfrak{b}\left(B_{2}\right)} \bigvee_{e \in \mathfrak{b}(a)}\{e\}=\mathfrak{b}\left(\mathfrak{b}\left(B_{1}\right) \vee_{\mathfrak{A}(P)} \mathfrak{b}\left(B_{2}\right)\right) \in \mathfrak{B}(P) \text {. }
\end{aligned}
$$

LEMMA 5.2. The meet-semilattice $2 \mathfrak{3}(P)$ is self-dual.

PRoof. Let $B_{1}, B_{2} \in \mathfrak{b}(P)$. If $B_{1} \leq_{\mathfrak{L}(P)} B_{2}$ then $B_{1} \leq_{\mathfrak{A}(P)} B_{2}$, and we see that $\mathfrak{b}\left(B_{1}\right) \geq_{\mathfrak{L}(P)}$ $\mathfrak{b}\left(B_{2}\right)$, by Lemma 4.1 .

Conversely, the relation $\mathfrak{b}\left(B_{1}\right) \geq_{\mathfrak{L}(P)} \mathfrak{b}\left(B_{2}\right)$ implies the relation $B_{1}=\mathfrak{b}\left(\mathfrak{b}\left(B_{1}\right)\right) \leq_{\mathfrak{Z}(P)}$ $B_{2}=\mathfrak{b}\left(\mathfrak{b}\left(B_{2}\right)\right)$, in view of Theorem 4.3 and Lemma 4.1.

Because the restriction map $\left.\mathfrak{b}\right|_{\mathfrak{B}(P)}$ is bijective, we see that it is an anti-automorphism of $\mathfrak{B}(P)$.

We now summarize the information of this section.

THEOREM 5.3. The poset $\mathfrak{2}(P)$ is a lattice with the least element $\hat{0}_{\mathfrak{B}(P)}=\varnothing \subset P$ and greatest element $\hat{1}_{\mathfrak{B}(P)}=\left\{\hat{0}_{P}\right\}$. The unique atom of $\mathfrak{b}(P)$ is $\mathfrak{b}\left(P^{\mathrm{a}}\right)$, and the unique coatom of $2 \mathfrak{B}(P)$ is $P^{\mathrm{a}}$. Moreover,

(i) the poset $\mathfrak{B}(P)$ is a meet-subsemilattice of the lattice $\mathcal{A}(P)$,

(ii) the lattice $2 \mathfrak{B}(P)$ is self-dual,

(iii) in the lattice $23(P)$ the operations of meet and join are determined as follows: for $B_{1}, B_{2} \in \mathfrak{2} \mathfrak{3}(P)$,

$$
\begin{gathered}
B_{1} \wedge_{\mathfrak{Z}(P)} B_{2}=B_{1} \wedge_{\mathfrak{A}(P)} B_{2}, \\
B_{1} \vee_{\mathfrak{S}(P)} B_{2}=\mathfrak{b}\left(\mathfrak{b}\left(B_{1}\right) \wedge_{\mathfrak{A}(P)} \mathfrak{b}\left(B_{2}\right)\right) .
\end{gathered}
$$

Proof. The only missing step is to prove (5.4), but the equality $B_{1} \vee_{\mathfrak{B}(P)} B_{2}=$ $\mathfrak{b}\left(\mathfrak{b}\left(B_{1}\right) \wedge_{\mathfrak{z}(P)} \mathfrak{b}\left(B_{2}\right)\right)$ immediately follows from the self-duality of the lattice $\mathfrak{2}(P)$, in view of the existence of its anti-automorphism $\left.\mathfrak{b}\right|_{\mathfrak{L}(P)}$. With the help of equality (5.3), we obtain (5.4).

We call the lattice $\mathfrak{B}(P)$ the lattice of blockers in the poset $P$. It follows immediately from the definition of the complementary map that its restriction $\left.\mathfrak{c}\right|_{\mathfrak{B}(P)}: \mathfrak{2} \mathfrak{B}(P) \rightarrow \mathfrak{C}(P)$, $B \mapsto \mathfrak{c}(B)$, is an isomorphism of $\mathfrak{B}(P)$ into the lattice $\mathfrak{C}(P)$.

\section{REFERENCES}

[1] R. Cordovil, K. Fukuda, and M. L. Moreira, Clutters and matroids, Discrete Math. 89 (1991), no. 2, 161-171. MR 92d:05040. Zbl 739.05019.

[2] J. Edmonds and D. R. Fulkerson, Bottleneck extrema, J. Combinatorial Theory 8 (1970), 299-306. MR 40\#8440. Zbl 218.05006.

[3] Z. Füredi, Matchings and covers in hypergraphs, Graphs Combin. 4 (1988), no. 2, 115-206. MR 89i:05214. Zbl 820.05051.

[4] C. Greene and D. J. Kleitman, The structure of Sperner k-families, J. Combinatorial Theory Ser. A 20 (1976), no. 1, 41-68. MR 53\#2695. Zbl 363.05006. 
[5] M. Grötschel, L. Lovász, and A. Schrijver, Geometric Algorithms and Combinatorial Optimization, 2nd ed., Algorithms and Combinatorics, vol. 2, Springer-Verlag, Berlin, 1993. MR 95e:90001. Zbl 837.05001.

[6] A. Lehman, A solution of the Shannon switching game, J. Soc. Indust. Appl. Math. 12 (1964), 687-725. MR 30\#3463. Zbl 137.38704.

[7] R. P. Stanley, Enumerative Combinatorics. Vol. 1, Cambridge Studies in Advanced Mathematics, vol. 49, Cambridge University Press, Cambridge, 1997. MR 98a:05001. Zbl 889.05001.

ANDrey O. MAtveev: Data-Center Company, RU-620034, P.O. BoX 5, EKaterinburg, RUSSIA

E-mail address: aomatveev@dc.ru 


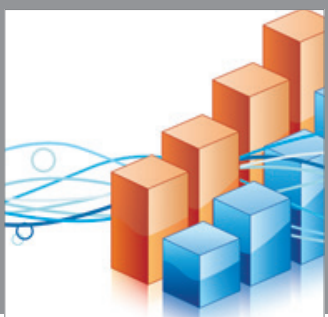

Advances in

Operations Research

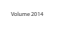

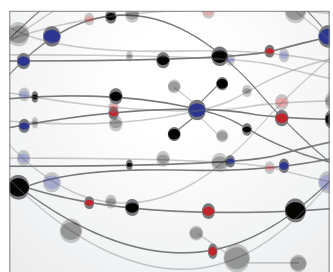

\section{The Scientific} World Journal
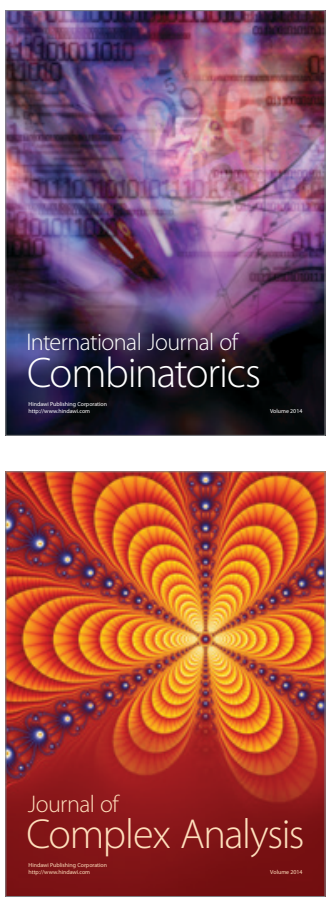

International Journal of

Mathematics and

Mathematical

Sciences
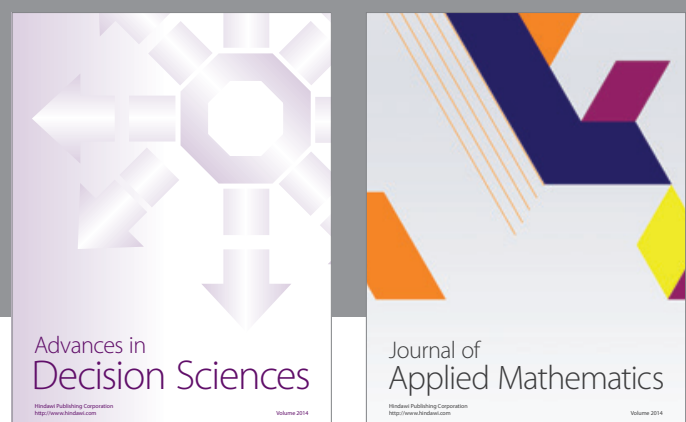

Journal of

Applied Mathematics
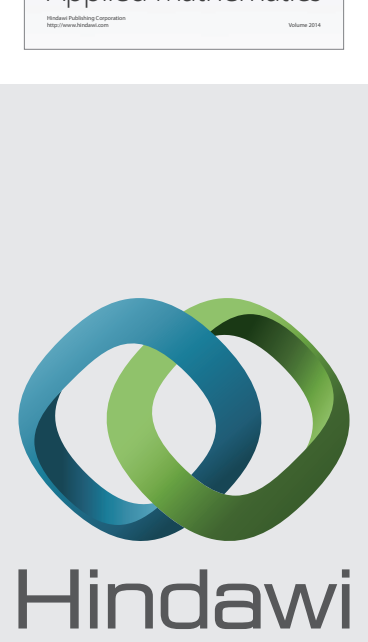

Submit your manuscripts at http://www.hindawi.com
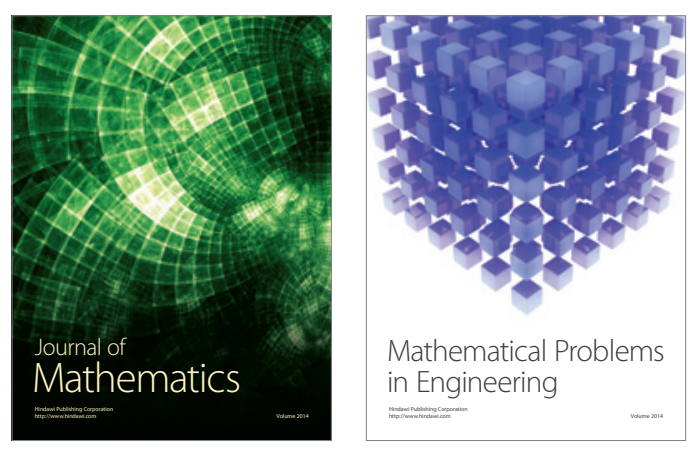

Mathematical Problems in Engineering
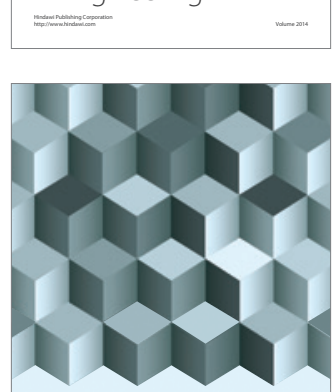

Journal of

Function Spaces
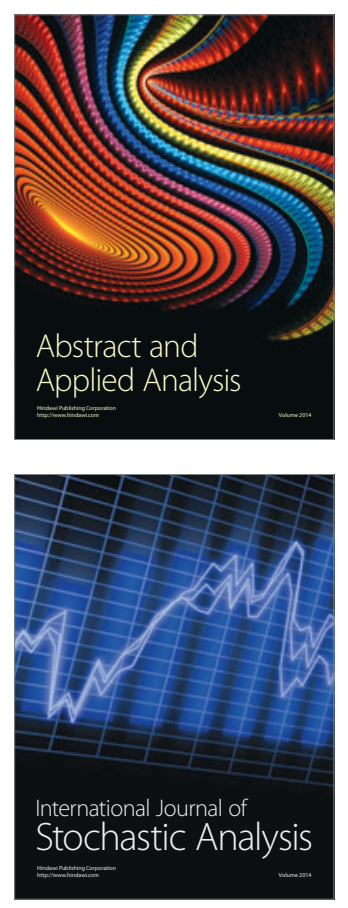

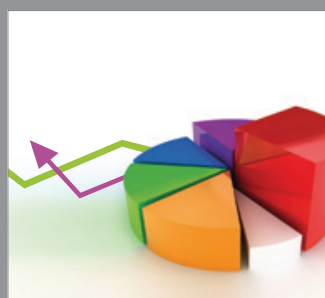

ournal of

Probability and Statistics

Promensencen
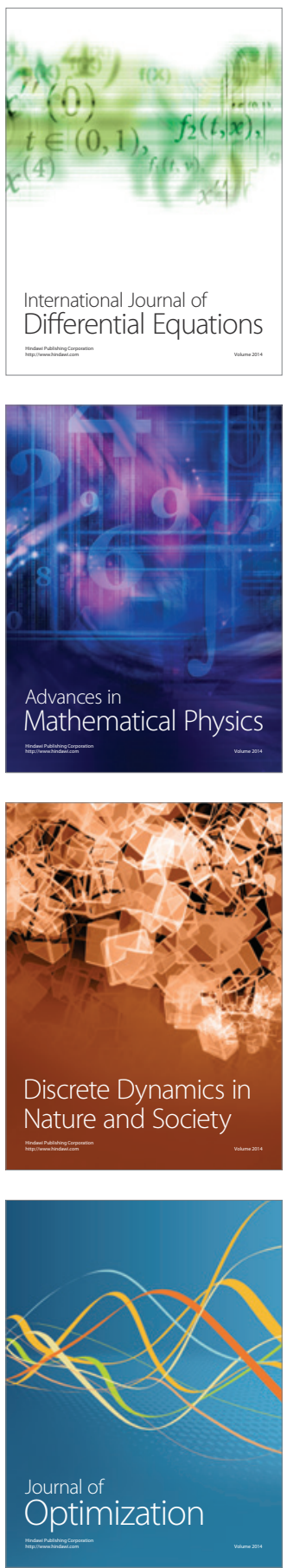